\title{
Coil Probe Dimension and Uncertainties During Measurements of Nonuniform ELF Magnetic Fields
}

\section{Martin Misakian}

National Institute of Standards and Technology, Gaithersburg, MD 20899-0001
Comparisons are made between the calculated average magnetic flux density for single-axis and three-axis circular coil probes and the calculated magnetic flux density at the center of the probes. The results, which are determined assuming a dipole magnetic field, provide information on the uncertainty associated with measurements of nonuniform extremely low frequency (ELF) magnetic fields produced by some electrical appliances and other electrical equipment.

Key words: appliance; coil probe; dipole field; magnetic field; measurement; measurement uncertainty; power frequency; residential; transportation systems; work place.

Accepted: January 28, 1993

\section{Introduction}

The concern in the mid 1970s regarding health effects from exposure to electric and magnetic fields in the vicinity of power lines has shifted in recent years to health effect concerns from exposure to power frequency magnetic fields in residences, the work place, and in transportation systems [1-3]. The magnetic fields in these environments can be highly nonuniform, particularly near electrical equipment such as motors, transformers and heating elements. This paper considers the difference between the calculated average magnetic flux density, $B_{\mathrm{av}}$, as determined using magnetic field meters with single-axis and three-axis circular coil probes, and the calculated magnetic flux density at the center of the probes, $B_{0}$ assuming the field is produced by a small loop of alternating current, i.e., a magnetic dipole. The magnetic dipole field is chosen as the relevant field because to a good approximation its geometry simulates the field geometry of many electrical appliances and equipment [4]. The difference between $B_{a v}$ and $B_{0}$ can be regarded as a source of measurement uncertainty because the center of the probe is normally considered the measurement location. While differences between $B_{\mathrm{av}}$ and $B_{0}$ will be small in many situations, e.g., near ground level in the vicinity of power lines where the field changes slowly, the difference can become significant in the highly nonuniform magnetic fields close to electrical equipment.

In this paper, two comparisons are made: (1) the maximum average magnetic field determined using a single-axis probe, $B_{\mathrm{avl}}$, with $B_{0}$ as a function of $r / a$ where $r$ is the distance between the magnetic dipole and the center of the probe, and $a$ is the radius of the probe, and (2) the resultant magnetic field determined using a three-axis probe with $B_{0}$ as a function of $r / a$. The resultant magnetic field, $B_{\text {av3, }}$, is defined as [5]

$$
B_{\mathrm{av3}}=\sqrt{B_{1}^{2}+B_{2}^{2}+B_{3}^{2}},
$$

where $B_{1}, B_{2}$, and $B_{3}$ are average magnetic field components as measured by three orthogonally oriented coil probes. 
Comparison (1) is made because maximum magnetic field values are sometimes measured, using single-axis field meters, to characterize the magnetic field [5,6]. However, for a given value of $r / a$, it will be seen that the difference between $B_{\text {av1 }}$ and $B_{0}$ will be a function of the orientation of the magnetic dipole relative to the probe. Because the relative orientation is not known during most measurements, what is of interest is the largest difference between $B_{\text {av1 }}$ and $B_{0}$ for a given value of $r / a$. This largest difference will be designated $\Delta B_{\max 1}$.

The quantity $\Delta B_{\max 1}$ is determined in the following way. The single-axis probe is rotated for fixed values of $r / a$ and the spherical coordinate, $\theta$, [Fig. 1(a)] until the largest average magnetic field, $B_{\text {av1 }}$, is found. This value of $B_{\text {av1 }}$ is compared with the magnetic field at the center of the probe, $B_{0}$ and the difference is recorded. The orientation of the magnetic dipole with respect to the probe is then varied by moving the probe to another location while keeping $r / a$ fixed, i.e., by changing $\theta$ in Fig. 1(a). The probe is rotated again until the largest average magnetic field, $B_{\mathrm{avl}}$, is found. $B_{\mathrm{av1}}$ is again compared with the magnetic field at the center of the probe, $B_{0}$, and the difference is recorded. This process is repeated for other dipole orientations (i.e., angle $\theta$ ) until the largest difference, $\Delta B_{\max 1}$, is found. An example of this process is shown in Sec. 3.1.

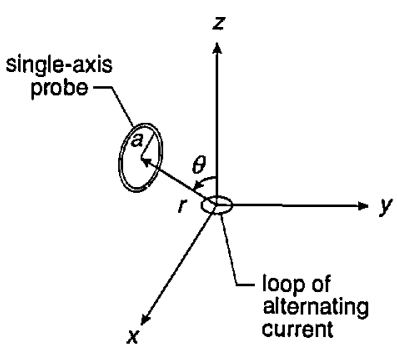

(a)

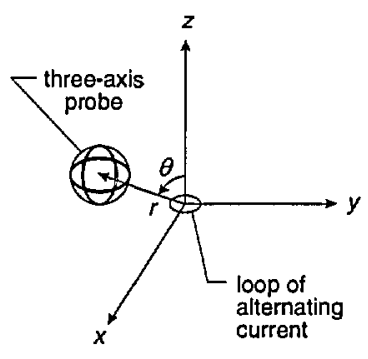

(b)
Fig. 1. (a) Single-axis and (b) three-axis circular coil probes in dipole magnetic field produced by small loop of current.

Comparison (2) is made as a three-axis probe is rotated about three axes parallel to the three Cartesian coordinates $x, y$, and $z$. The difference between $B_{\text {av3 }}$ and $B_{0}$ will vary as a function of rotation angle, but what will be of interest again is the largest difference, $\Delta B_{\max 3}$, for a given $r / a$. Also as for comparison (1), because the relative orientations of the magnetic dipole and the three-axis probe will be unknown in most measurement situations, $B_{\text {av3 }}$ will be examined as a function of $r / a$ and the spherical coordinate, $\theta$, in order to determine the largest difference, $\Delta B_{\max 3}$.

\section{Expressions for Average Magnetic Flux Density}

In the derivations given below, it is assumed that the cross sectional area of the wire in the coil probes and the opposing magnetic field produced by current induced in the probes are negligible. In addition, we assume for the three-axis probe that the three orthogonally oriented coils have circular cross sections of equal area. These assumptions either can be met in practice or can be taken into account via a calibration process.

\subsection{Single-Axis Probe}

The average magnetic flux density, $B_{\mathrm{av}}$, for a single coil probe with cross sectional area $A$ is given by

$$
B_{\mathrm{av}}=\frac{1}{A} \iint_{A} \boldsymbol{B} \cdot \hat{n} \mathrm{~d} A,
$$

where $\mathrm{d} A$ is an element of probe area, $\hat{n}$ is a unit vector perpendicular to $A$, and $B$ is the magnetic flux density. In spherical coordinates, the magnetic flux density for a small current loop of radius $b$ is [7]

$$
\boldsymbol{B}=\frac{\mu_{0} I b^{2}}{2 r^{3}} \cos \theta \hat{u}_{r}+\frac{\mu_{0} I b^{2}}{4 r^{3}} \sin \theta \hat{u}_{\theta},
$$

where $\mu_{0}$ is the permeability of vacuum, $I$ is the alternating current, and $\hat{u}_{r}$ and $\hat{u}_{\theta}$ are unit vectors in the directions of increasing $r$ and $\theta$, respectively. The assumption is made that $b<<r$ and the sinusoidal time dependence has been suppressed. The magnitude of the vector $\boldsymbol{B}$ given by Eq. (3) is $B_{0}$.

For our purposes, it is convenient to express $B$ in terms of Cartesian coordinates. This is accomplished by using the following relations between spherical and Cartesian unit vectors and coordinates [8] in Eq. (3):

$$
\begin{aligned}
& \hat{u}_{r}=\hat{\imath} \sin \theta \cos \phi+\hat{\jmath} \sin \theta \sin \phi+\hat{k} \cos \theta \\
& \hat{u}_{\mathrm{B}}=\hat{\imath} \cos \theta \cos \phi+\hat{\jmath} \cos \theta \sin \phi-\hat{k} \sin \theta \\
& x=r \sin \theta \cos \phi \\
& y=r \sin \theta \sin \phi \\
& z=r \cos \theta .
\end{aligned}
$$

After some algebra, $\boldsymbol{B}$ can be expressed as

$$
\boldsymbol{B}=\hat{\imath} \frac{3 C x z}{2 r^{5}}+\hat{\jmath} \frac{3 C y z}{2 r^{5}}+\hat{k} \frac{C}{2 r^{3}}\left(\frac{3 z^{2}}{r^{2}}-1\right),
$$


where $r=\sqrt{x^{2}+y^{2}+z^{2}}$ and $C$ is the constant $\mu_{0} I b^{2} / 2$.

To obtain an expression for $B_{\mathrm{av}}$, we consider without loss of generality a probe with its center at $x=x_{0}, y=0$, and $z=z_{0}$ as shown in Fig. 2. We restrict the orientation of the probe so that its area is bisected by the $x-z$ plane and first consider rotations of the probe about an axis parallel to the $y$-axis, i.e., the $y^{\prime}$-axis shown in Fig. 2. For these conditions, the area of the coil probe, $A$, will be part of the surface given by the equation

$$
z=m_{\alpha}\left(x-x_{0}\right)+z_{0}
$$

where $\alpha$ is the angle of rotation, $m_{\alpha}=\tan \alpha$, $x_{0}=r \sin \theta$, and $z_{0}=r \cos \theta$. The rotation of the probe corresponds to the rotation of this surface about the $y^{\prime}$-axis, i.e., changing the slope of the surface $\left(m_{\alpha}\right)$ described by Eq. (6). It should be noted that the angle of rotation, $\alpha$, shown in Fig. 2 is in the negative direction.

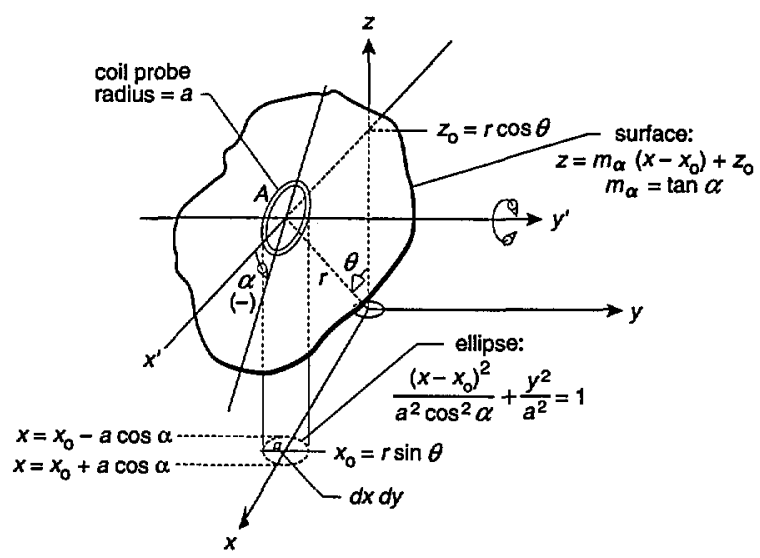

Fig. 2. Circular coil probe shown as part of a surface described by the equation $z=m_{\alpha}\left(x-x_{0}\right)+z_{0}$. The rotation of the probe corresponds to changing the slope of the surface, $m_{\alpha}$. The projection of the probe cross sectional area onto the $x-y$ plane will be an ellipse for $\alpha \neq 0$. The range of $\alpha$ is $-90^{\circ}<\alpha<90^{\circ}$.

The unit vector perpendicular to the probe surface, $\hat{n}$, is found by first taking the gradient [9] of the surface given by Eq. (6), $\nabla F(x, z)$, where $F(x, z)=z-m_{\alpha}\left(x-x_{0}\right)-z_{0}$ and normalizing it to unit value. This leads to

$$
\hat{n}=\left(-m_{\alpha} \hat{\imath}+\hat{k}\right) / \sqrt{m_{\alpha}^{2}+1}
$$

The element of area, $\mathrm{d} A$, is [10]

$$
\mathrm{d} A=\sqrt{\left(\frac{\delta z}{\delta x}\right)^{2}+\left(\frac{\delta z}{\delta y}\right)^{2}+1} \mathrm{~d} x \mathrm{~d} y=\sqrt{m_{\alpha}^{2}+1} \mathrm{~d} x \mathrm{~d} y .
$$

where $\mathrm{d} x \mathrm{~d} y$ is an element of area in the $x-y$ plane bounded by the projection of the probe cross section onto the $x-y$ plane (Fig.2).

Combining Eqs. (2), (5), (7), and (8), the expression for $B_{\text {av }}$ becomes

$B_{\mathrm{av}}=\frac{C}{2 \pi a^{2}} \int_{x} \int_{y}\left\{\frac{-3 x z m_{\alpha}}{r^{5}}+\frac{1}{r^{3}}\left(\frac{3 z^{2}}{r^{2}}-1\right)\right\} \mathrm{d} x \mathrm{~d} y$.

By substituting for $z$ in Eq. (9) using Eq. (6), the integrand becomes a function of $x$ and $y$. The integration is first carried out analytically [11] over the variable $y$ with (from Fig.2)

$$
\begin{gathered}
-\sqrt{a^{2}-\left(\left(x-x_{0}\right) / \cos \alpha\right)^{2}} \leq y \leq \\
\sqrt{a^{2}-\left(\left(x-x_{0}\right) / \cos \alpha\right)^{2}} .
\end{gathered}
$$

The resulting expression for $B_{\mathrm{av}}$ is

$$
\begin{gathered}
B_{\mathrm{av}}=-\frac{C}{\pi a^{2}} \int \mathrm{d} x\left(m_{a} x P+P^{2}\right) \\
\left\{\frac{\sqrt{a^{2}-\left(\frac{x-x_{0}}{\cos \alpha}\right)^{2}}}{\left(x^{2}+Q^{2}\right)\left(x^{2}+Q^{2}+a^{2}-\left(\frac{x-x_{0}}{\cos \alpha}\right)^{2}\right)^{3 / 2}}+\right. \\
\left.\frac{2 \sqrt{a^{2}-\left(\frac{x-x_{0}}{\cos \alpha}\right)^{2}}}{\left(x^{2}+Q^{2}\right)^{2}\left(x^{2}+Q^{2}+a^{2}-\left(\frac{x-x_{0}}{\cos \alpha}\right)^{2}\right)^{1 / 2}}\right\}- \\
\frac{C}{\pi a^{2}} \int \mathrm{d} x \frac{\left(x^{2}-\left(\frac{x-x_{0}}{\cos \alpha}\right)^{2}\right.}{\left(x^{2}\right)\left(x^{2}+Q^{2}+a^{2}-\left(\frac{x-x_{0}}{\cos \alpha}\right)^{2}\right)^{1 / 2}}
\end{gathered}
$$


where $P=\left(z_{0}-m_{a} x_{0}\right)$ and $Q^{2}=\left(m_{a} x+P\right)^{2}$.

The integration over $x$ is then performed numerically using Simpson's Rule with the limits of integration given by (Fig. 2)

$$
x_{0}-a \cos \alpha \leq x \leq x_{0}+a \cos \alpha \text {, }
$$

where $\alpha$ is restricted to $-90^{\circ}<\alpha<90^{\circ}$.

$B_{\mathrm{av}}$ is evaluated for fixed values of $\theta$ and $r / a$ as $\alpha$ is varied until a maximum average flux density, $B_{\text {av1 }}$, is found. $B_{\text {av1 }}$ is then compared with $B_{0}$. As noted earlier, the process is repeated for the same $r / a$ but different values of $\theta$ until the largest difference, $\Delta B_{\max }$, is determined. Because we are seeking the maximum value of $B_{\mathrm{av}}$, we do not consider further rotations of the probe because once $B_{\text {av1 }}$ is found, additional rotations are expected to lead to smaller values of $B_{\mathrm{av}}$. This is most readily seen at $\theta$ equal to $0^{\circ}$ and $90^{\circ}$ for all values of $r / a . B_{\text {av1 }}$ occurs at $\alpha=0^{\circ}$ and rotating the probe further results in smaller values of $B_{\text {av }}$.

\subsection{Three-Axis Probe}

In this section, expressions are developed for the average magnetic flux density for each coil of a three-axis probe as the probe is rotated about axes which are parallel to the $x-, y$-, and $z$-axes. Afterwards, for fixed values of $\theta$ and $r / a$, the average magnetic field values from the three orthogonally oriented probes are combined according to Eq. (1) to obtain $B_{\text {av3 } 3}$ which is then compared with $B_{0}$. As before, the process is repeated for different values of $\theta$ until the maximum difference between $B_{\text {av3 }}$ and $B_{0}, \Delta B_{\max 3}$, is found.

It is noted that combinations of rotations about the different axes will not be possible using the expressions that are developed. That is, it will not be possible to calculate $B_{\text {av }}$ following rotations about two or three axes. This represents a limitation on the results and prevents us from learning whether there are significant effects on the value of $\Delta B_{\max 3}$ due to multiple rotations. Nevertheless, the departures from $B_{0}$ that are determined from rotations about each of the three axes will let us know what differences are possible as a function of $r / a$.

Figure 3 shows the orientation of the probe with respect to the magnetic dipole before rotations about each axis are considered. As in the previous section, the center of the probe is located at $x=x_{0}$, $y=0$, and $z=z_{0}$. The individual probes are labelled $\mathrm{P} 1, \mathrm{P} 2$, and $\mathrm{P} 3$, and initially $\mathrm{P} 1$ lies in the $x^{\prime}-y^{\prime}$ plane, $\mathrm{P} 2$ is in the $y^{\prime}-z^{\prime}$ plane, and $\mathrm{P} 3$ is in the $x^{\prime}-z^{\prime}$ plane. When rotations are performed about the $x^{\prime}$-axis (rotation angle $\beta$ ), the angle that P2 makes with the magnetic field remains unchanged. Similarly, rotations about the $y^{\prime}$-axis (rotation angle $\alpha$ ) and $z^{\prime}$-axis (rotation angle $\gamma$ ) leave $\mathrm{P} 3$ and P1, respectively, "unchanged." This means that for constant values of $r / a$ and $\theta$, the average flux density values for these "fixed" probes remain constant as rotations of the probe occur.

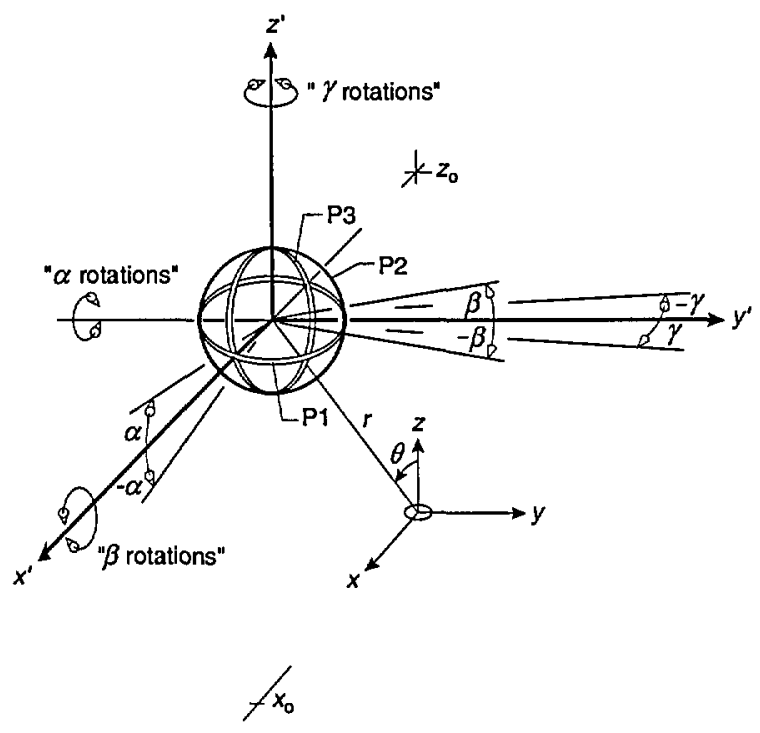

Fig. 3. Geometry for three-axis probe with center of probe in $x-z$ plane. Varying the angle $\alpha$ result in rotations of probes P1 and P2 about the $y^{\prime}$-axis (" $\alpha$ rotations") while orientation of probe $\mathrm{P} 3$ with respect to the dipole remains unchanged. Varying the angle $\beta$ results in rotations of probes $P 1$ and $P 3$ about the $x$-axis (" $\beta$ rotations") while orientation of probe $P 2$ remains unchanged. Varying the angle $\gamma$ results in rotations of probes P2 and $\mathrm{P} 3$ about the $z$ '-axis (" $\gamma$ rotations") while orientation of probe $P 1$ remains unchanged.

We begin the derivation for the three-axis probe by noting that part of the problem has already been solved in the previous section. That is, the expression for $B_{\mathrm{av}}$ following rotations about the $y^{\prime}$-axis ( $\alpha$ rotations) is given by Eq. (10). This expression is used to calculate the average flux density for probes $\mathrm{P} 1$ and $\mathrm{P} 2$ by considering pairs of the angle $\alpha$ which differ by $90^{\circ}$. The average flux density for the third probe, $P 3$, is zero for this case because no component of the magnetic field is perpendicular to the area of the probe for any value of $\alpha$ or $\theta$.

The derivations for $B_{\text {av }}$ following $\beta$ or $\gamma$ rotations parallel the derivation for the $\alpha$ rotations. Examining the case of $\beta$ rotations first, and referring to Fig. 4, it can be seen that the probe area, $A$, is part of the surface given by the equation

$$
z=m_{\beta} y,+z_{0},
$$




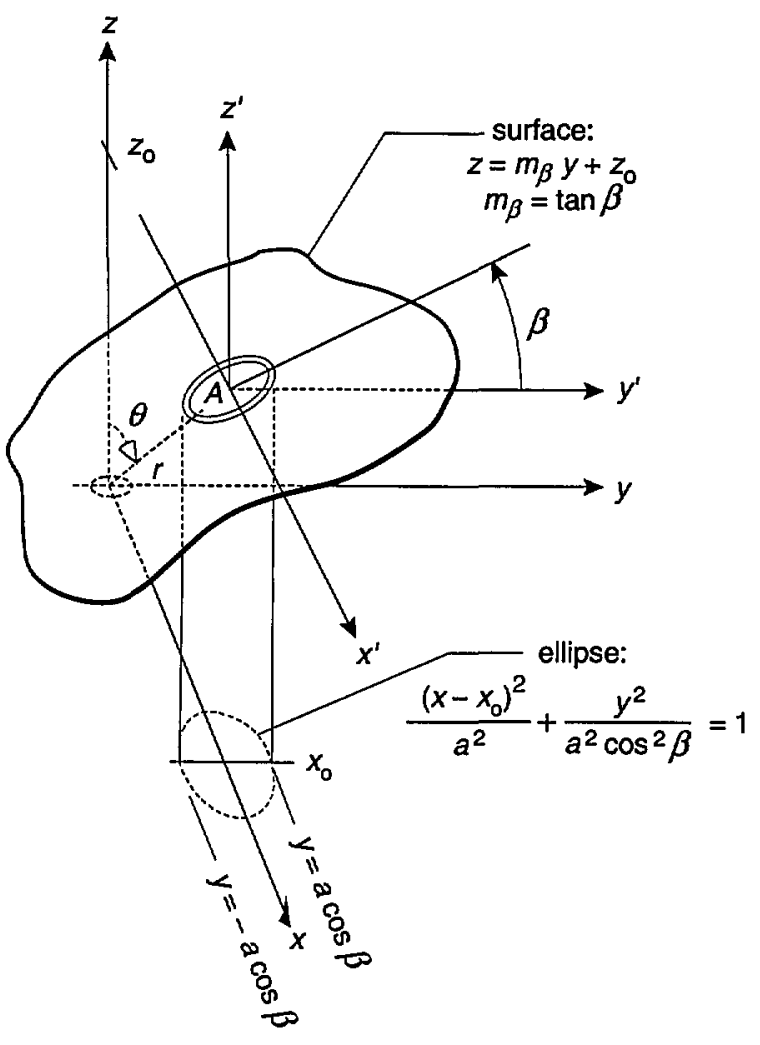

Fig. 4. Circular coil probe shown as part of a surface described by the equation $z=m_{\beta} y+z_{0}$. The rotation of the probe corresponds to changing the slope of the surface, $m_{\beta}$. The projection of the probe cross sectional area onto the $x-y$ plane will be an ellipse for $\beta \neq 0$. The range of $\beta$ is $-90^{\circ}<\beta<90^{\circ}$.

where $m_{\beta}=\tan \beta$ and $z_{0}=r \cos \theta$. The rotation of the probe corresponds to rotation of this surface about the $x^{\prime}$-axis or alternatively, changing its slope, $m_{\beta}$. The unit vector normal to this surface is

$$
\hat{n}=\left(-m_{\beta} \hat{\jmath}+\hat{k}\right) / \sqrt{m_{\beta}^{2}+1},
$$

and the element of area, $\mathrm{d} A$, is

$$
\mathrm{d} A=\sqrt{\left(\frac{\delta z}{\delta x}\right)^{2}+\left(\frac{\delta z}{\delta y}\right)^{2}+1} \mathrm{~d} x \mathrm{~d} y=\sqrt{m_{\beta}^{2}+1} \mathrm{~d} x \mathrm{~d} y .
$$

Combining Eqs. (2), (5), (12), and (13), the expression for the average flux density following $\beta$ rotations is

$$
\begin{gathered}
B_{\text {av } \beta}=\frac{1}{\pi a^{2}} \int_{y} \int_{x} B \cdot \hat{n} \mathrm{~d} A= \\
-\frac{3 C m_{\beta}}{2 \pi a^{2}} \int_{y} \int_{x} \frac{y z}{r^{5}} \mathrm{~d} x \mathrm{~d} y+ \\
\frac{C}{2 \pi a^{2}} \int_{y} \int_{x}\left(\frac{3 z^{2}}{r^{5}}-\frac{1}{r^{3}}\right) \mathrm{d} x \mathrm{~d} y .
\end{gathered}
$$

Substituting for $z$ in Eq. (14) using Eq. (11), the integrands become, recalling that $r=\sqrt{x^{2}+y^{2}+z^{2}}$, a function of $x$ and $y$. The integration is first carried out analytically over the variable $x$ with (Fig. 4)

$$
x_{0}-\sqrt{a^{2}-(y / \cos \beta)^{2}} \leq x \leq x_{0}+\sqrt{a^{2}-(y / \cos \beta)^{2}} .
$$

The $y$-integration is performed numerically with the limits of integration given by (Fig. 4)

$$
-a \cos \beta \leq y \leq a \cos \beta,
$$

where $\beta$ is restricted to $-90^{\circ}<\beta<90^{\circ}$.

Equation (14) is used to calculate the average flux density for probes P1 and P3 by considering pairs of the angle $\beta$ which differ by $90^{\circ}$. The average flux density from the remaining probe, $P 2$, remains constant during the $\beta$ rotations and is determined from the expression for average flux density following $\gamma$ rotation $(\gamma=0)-$ which is developed below. The reader is cautioned that during the numerical integration over the variable $y$, the denominator in the integrand vanishes for $y=0$ when $\theta=90^{\circ}$.

In deriving the expression for average flux density following $\gamma$ rotations, we note as shown in Fig. 5 that the probe area, $A$, is part of the surface given by the equation

$$
x=m_{\gamma} y+x_{0},
$$

where $m_{\gamma}=\tan \gamma$ and $x_{0}=r \sin \theta$. The rotation of the probe corresponds to rotation of this surface about the $z^{\prime}$-axis, i.e., changing the slope of the surface, $m_{\gamma}$. The unit vector normal to this surface is

$$
\hat{n}=\left(\hat{\imath}-m_{\gamma} \hat{\jmath}\right) / \sqrt{m_{\gamma}^{2}+1},
$$


and the element of area, $\mathrm{d} A$, is

$$
\mathrm{d} A=\sqrt{\left(\frac{\delta x}{\delta y}\right)^{2}+\left(\frac{\delta x}{\delta z}\right)^{2}+1} \mathrm{~d} y \mathrm{~d} z=\sqrt{m_{\gamma}^{2}+1} \mathrm{~d} y \mathrm{~d} z .
$$

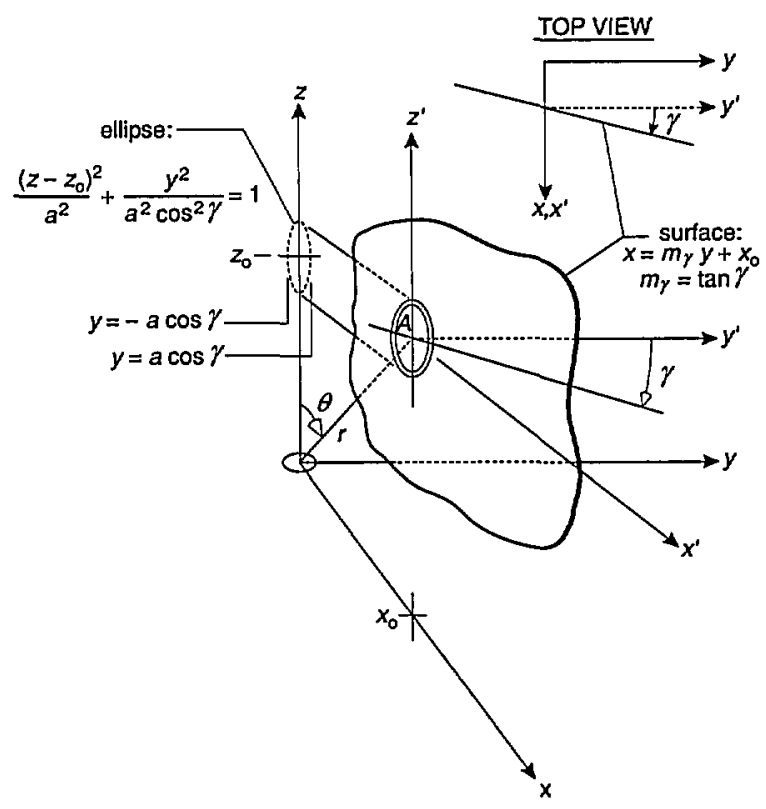

Fig. 5. Circular coil probe shown as part of a surface described by the equation $x=m_{\gamma} y+x_{0}$. The rotation of the probe corresponds to changing the slope of the surface, $m_{\gamma}$. The projection of the probe cross sectional area onto the $y-z$ plane will be an ellipse for $\gamma \neq 0$. The range of $\gamma$ is $-90^{\circ}<\gamma<90^{\circ}$.

It should be noted that the projection of the probe's cross sectional area, unlike the previous two cases, is onto the $y-z$ plane for $\gamma$ rotations (the projections for $\alpha$ and $\beta$ rotations were onto the $x-y$ plane) and this fact affects the partial derivatives in the expression for $\mathrm{d} A$, Eq. (17).

From Eqs. (2), (5), (16), and (17), the expression for average flux density following $\gamma$ rotations is

$$
\begin{aligned}
& B_{\mathrm{av} \gamma}=\frac{1}{\pi a^{2}} \int_{y} \int_{z} B \cdot \hat{n} \mathrm{~d} A= \\
& \frac{3 C}{2 \pi a^{2}} \int_{y} \int_{z}\left(\frac{x z}{r^{5}}-\frac{m_{y} y z}{r^{5}}\right) \mathrm{d} z \mathrm{~d} y .
\end{aligned}
$$

By substituting for $x$ in Eq. (18) using Eq. (15), the integrand becomes a function of $y$ and $z$. The integration is first carried out analytically over the variable $z$ with (Fig. 5) $z_{0}-\sqrt{a^{2}-(y / \cos \gamma)^{2}} \leq z \leq z_{0}+\sqrt{a^{2}-(y / \cos \gamma)^{2}}$.

The $y$-integration is performed numerically with the limits of integration given by

$$
-a \cos \gamma \leq y \leq a \cos \gamma,
$$

where $\gamma$ is restricted to $-90^{\circ}<\gamma<90^{\circ}$.

Equation (18) is used to calculate the average flux density for probes $\mathrm{P} 2$ and $\mathrm{P} 3$ by considering pairs of the angle, $\gamma$, which differ by $90^{\circ}$. The average flux density from the remaining probe, P1, remains constant during the $\gamma$ rotations and is calculated from the expression for average flux density for $\alpha$ rotations [Eq. (10)] with $\alpha$ set equal to zero.

\section{Results of Calculations}

\section{1 $\Delta B_{\max 1}$ for Single-Axis Probe}

Using Eq. (10) and following the procedure described after Eq. (1), values of the maximum average magnetic field, $B_{\text {avs }}$ for fixed values of $r / a$ and $\theta$ were calculated and compared with the corresponding value of $B_{0}$. Figure 6 shows the differences in percent between $B_{\text {av1 }}$ and $B_{0}$ for $r / a=3$ and for representative values of $\theta$ between $0^{\circ}$ and $90^{\circ}$ (because of symmetry arguments, one can infer the corresponding percentages for $\theta$ between $90^{\circ}$ and $180^{\circ}$ ). The largest difference, $\Delta B_{\max }$, is $-14.6 \%$ and occurs when the single-axis probe is located along the axis of the magnetic dipole, i.e., the $z$-axis. The negative difference between $B_{\text {av1 }}$ and $B_{0}$ decreases as $\theta$ increases and turns positive near $\theta=90^{\circ}$. This pattern also occurs for other values of $r / a$ greater than 3. Figure 6 also shows the largest negative and positive differences in percent for $r / a$ equal to 5,8 , 10 , and 12. The largest negative differences must be considered part of the measurement uncertainty when the probe-dipole geometry is unknown, which will be the case for example when magnetic field measurements are performed near many appliances. A tabulation of $\Delta B_{\max }$, as a function of $r / a$ is given in Table 1.

The calculations are not carried out for large values of $r / a$ because the accuracy requirements for magnetic field measurements near appliances and other electrical equipment either have not been set or are not great. For example, the uncertainty tentatively allowed during calibration of magnetic field meters used for measuring magnetic fields near visual display terminals is $\pm 5 \%$ [12]. 


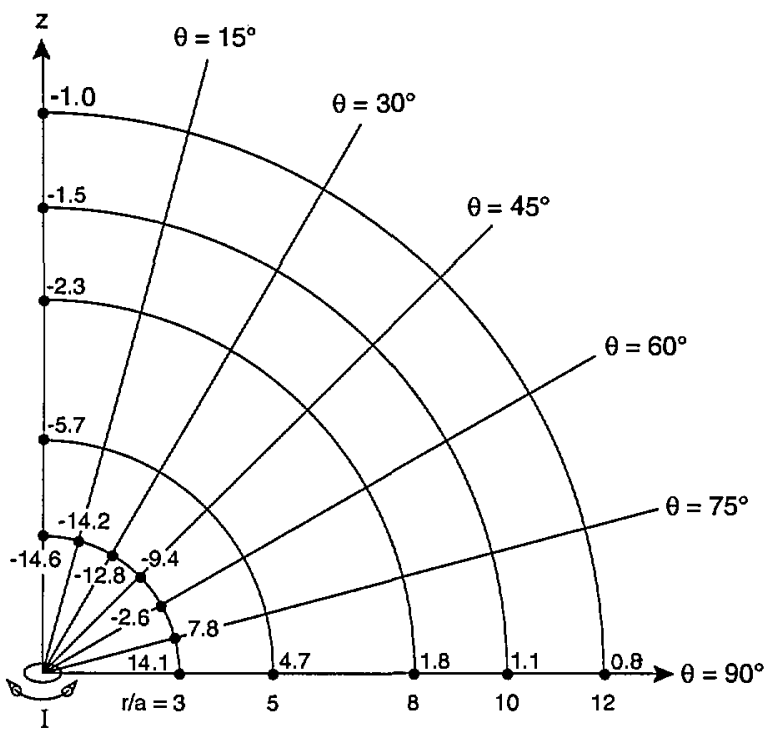

Fig. 6. Differences between values of $B_{\text {av1 }}$ and $B_{0}$, in percent, for different locations of single-axis probe relative to magnetic dipole which is aligned along the $z$-axis. For a given value of $r / a$, where $r / a$ is $\geqslant 3$, the largest difference, $\Delta B_{\operatorname{maxl}}$, is negative and occurs when the probe is located along the $z$-axis.

Table 1. Values of $\Delta B_{\max 1}$ (single-axis probe) and $\Delta B_{\max 3}$ (three-axis probe) as a function of normalized distance (r/a) from magnetic dipole

\begin{tabular}{rrc}
\hline \hline rla & $\Delta B_{\operatorname{maxl}}(\%)$ & $\Delta B_{\max 3}(\%)$ \\
\hline 3 & -14.6 & -19.6 \\
4 & -8.7 & -10.8 \\
5 & -5.7 & -6.9 \\
6 & -4.0 & -4.8 \\
7 & -3.0 & -3.5 \\
8 & -2.3 & -2.7 \\
9 & -1.8 & -2.1 \\
10 & -1.5 & -1.7 \\
11 & -1.2 & -1.4 \\
12 & -1.0 & -1.2 \\
13 & -0.9 & -1.0 \\
14 & -0.8 & -0.9 \\
15 & -0.7 & -0.8 \\
\hline
\end{tabular}

\section{2 $\Delta B_{\max 3}$ for Three-Axis Probe}

The differences between $B_{\text {av3 }}$ and $B_{0}$ are considered in three steps. First, values of $B_{\text {av3 }}$ are calculated following $\alpha$ rotations using Eq. (10) and compared with $B_{0}$ for fixed values of $r / a$ and representative values of $\theta$ between $0^{\circ}$ and $90^{\circ}$. As noted earlier, the largest difference between $B_{\text {av3 }}$ and $B_{0}$ at each point, a "local maximum difference," is recorded. In the discussion that follows, the "local maximum difference," will be referred to simply as the "difference." The above procedure is repeated for $\beta$ and $\gamma$ rotations.

The differences between $\beta_{\text {ays }}$ and $\beta_{0}$ following $\alpha$ rotations are plotted in Fig. 7 for $r / a$ equal to 3,5 , 8,10 , and 12. The numbers in Fig. 7 represent differences in percent. The pattern observed for all values of $r / a$ is that the difference at a point following $\alpha$ rotation is always negative and becomes more negative as $\theta$ increases to $90^{\circ}$.

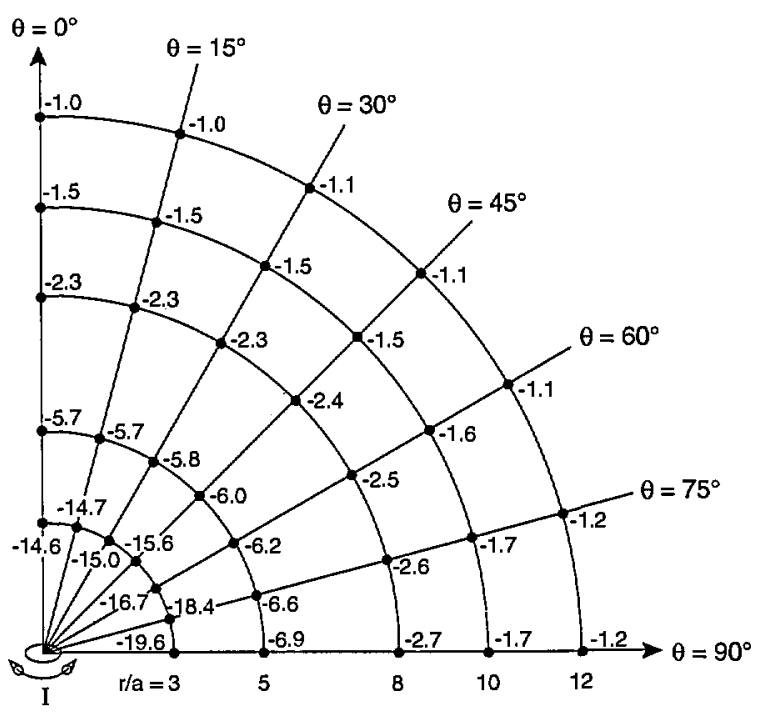

Fig. 7. Differences between values of $B_{\mathrm{av} 3}$ and $B_{0}$ in percent, for different locations of three-axis probe relative to magnetic dipole, following $\alpha$ rotations. The differences are always negative and the greatest difference following $\alpha$ rotations occurs for $\theta=90^{\circ}$.

When the difference calculations are performed for $\beta$ rotations using Eq. (14) and Eq. (18) ( $\gamma$ set equal to zero), a different pattern emerges. The differences between $B_{\mathrm{av} 3}$ and $B_{0}$ are observed to change in sign at different points as shown in Fig. 8. By comparing the results in Figs. 7 and 8, it can be seen that, except for $\theta=0$, the differences following $\beta$ rotations are all less than the corresponding (i.e., same $r / a$ and $\theta$ values) differences following $\alpha$ rotations. Although not indicated in Fig. 8 calculations show that for a given value of $r / a$ $\geq 3$, the differences between $B_{\text {av }}$ and $B_{0}$ following $\beta$ rotations for all values of $\theta$ are less than the difference following $\alpha$ rotations when $\theta=90^{\circ}$.

Similar results occur following $\gamma$ rotations when $B_{a v 3}$ is calculated using Eq. (18) and Eq. (10) ( $\alpha$ set equal to zero) and is compared with $B_{0}$. Once again the differences between $B_{\text {av3 }}$ and $B_{0}$ change in sign as shown in Fig. 9. Also, by comparing results in Figs. 7 and 9 , it is seen that, except for $\theta=0^{\circ}$, the 


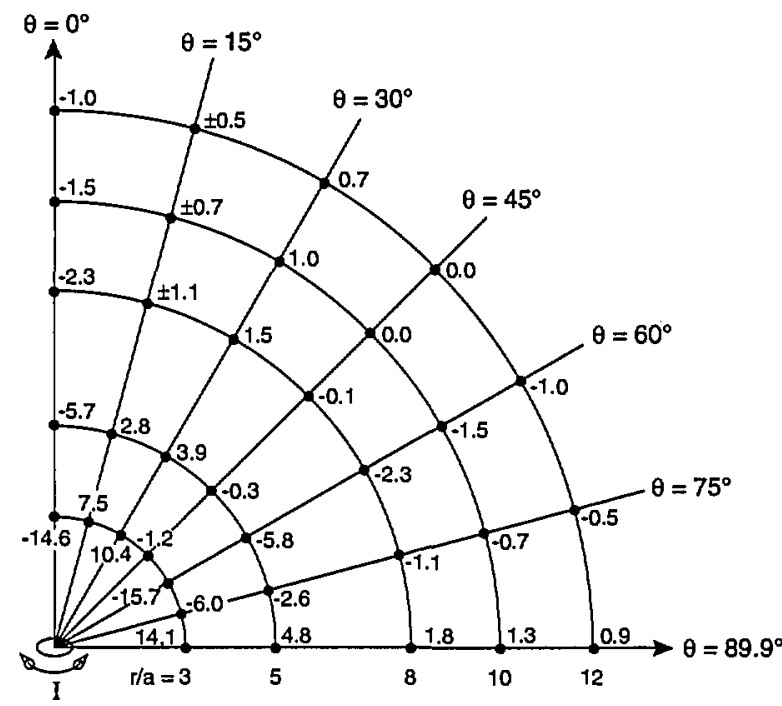

Fig. 8. Differences between values of $B_{\text {av3 }}$ and $B_{0}$ in percent, for different locations of three-axis probe relative to magnetic dipole, following $\beta$ rotations. The differences vary in sign depending on angle $\theta$. For $\theta=15^{\circ}$, there are several cases (indicated with the \pm sign) for which the largest negative and positive differences are equal in magnitude.

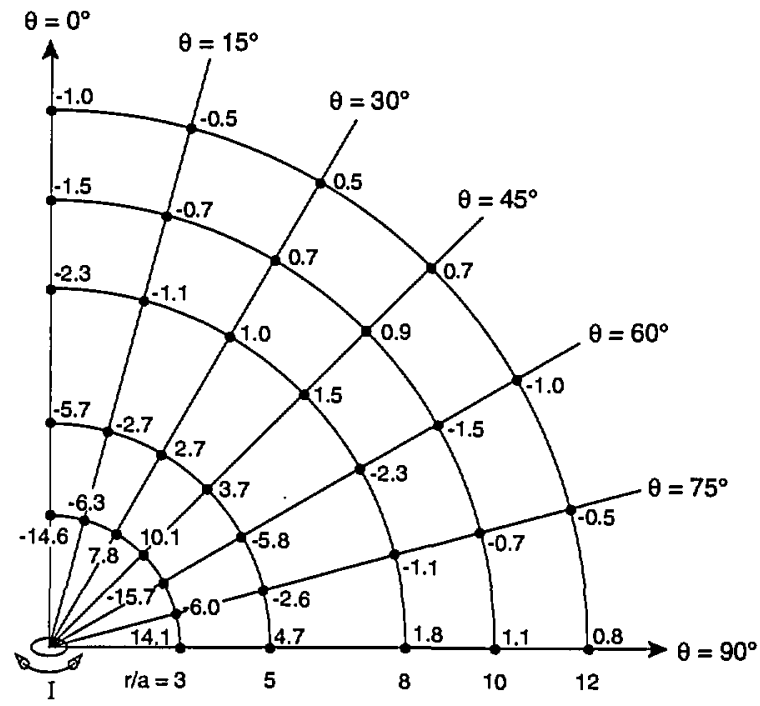

Fig. 9. Differences between values of $B_{\mathrm{av} 3}$ and $B_{0}$ in percent, for different locations of three-axis probe relative to magnetic dipole, following $\gamma$ rotations. The differences vary in sign depending on angle $\theta$.

differences following $\gamma$ rotations are all less than the corresponding differences following $\alpha$ rotations. As for the case of $\beta$ rotations, calculations show that for a given value of $r / a \geq 3$, the largest difference following $\gamma$ rotations will always be less than the difference following $\alpha$ rotations when $\theta=90^{\circ}$. Therefore, of the three types of three-axis probe rotations considered, the greatest difference between $B_{\text {av3 }}$ and $B_{0}$ can be found following $\alpha$ rotations and $\Delta B_{\max 3}$ occurs when $\theta=90^{\circ}$ for a given value of $r / a$. Table 1 provides a listing of $\Delta B_{\max 3}$ values as a function of $r / a$.

\section{Discussion of Results}

Once it has been decided what constitutes an acceptable level of uncertainty during magnetic field measurements near electrical equipment, the information in Table 1 should be considered when taking into account the various sources of measurement uncertainty. For example, if maximum magnetic fields at a distance $r$ from appliances are to be measured with a total uncertainty of less than $\pm 10 \%$, magnetic field meters with probes having radii $a$ such that $r / a \approx 3$ would immediately be considered unsuitable. Field meters with single-axis probes having radii such that $r / a=5$ would be suitable if all other sources of uncertainty (e.g., calibration process, frequency response) amounted to about $8 \%$ or less, i.e., $\sqrt{5.7^{2}+8^{2}}=9.8$, where 5.7 is taken from Table 1 for $r / a=5$.

The measurement uncertainties associated with using three-axis probes are less clear because we have considered only separate rotations about three axes to obtain the values of $\Delta B_{\max 3}$. The percentage differences in Table 1 indicate what uncertainties can occur but they may not be the largest uncertainties due to the averaging effects of the probe. However, until calculations can be devised which consider more complex rotations of three-axis probes, the $\Delta B_{\max 3}$ values in Table 1 can serve as a rough guide when deciding what are acceptable probe dimensions.

\section{Acknowledgments}

This work was performed in the Electricity Division, Electronics and Electrical Engineering Laboratory, National Institute of Standards and Technology, Technology Administration of the U.S. Department of Commerce. Support was received from the Office of Energy Management of the U.S. Department of Energy.

\section{References}

[1] N. Wertheimer and E. Leeper, Am. J. Epidemiol. 109, 273 (1979). 
[2] D. A. Savitz and E. E. Calle, J. Occup. Med. 29, 47 (1987).

[3] Abstracts W1-W7, First World Congress for Electricity and Magnetism in Biology and Medicine, Lake Buena Vista, FL (1992), pp. 65-67.

[4] D. L. Mader and S. B. Peralta, Bioelectromagnetics 13, 287 (1992).

[5] IEEE Magnetic Fields Task Force, A Protocol for Spot Measurements of Residential Power Frequency Magnetic Fields, IEEE Trans. Power Delivery, in press.

[6] J. R. Gauger, IEEE Trans. Power Appar. Syst. PAS-104, 2436 (1985).

[7] D. Corson and P. Lorrain, Introduction to Electromagnetic Fields and Waves, W. H. Freeman, San Francisco, CA (1962), p. 210.

[8] K. R. Symon, Mechanics, Addison-Wesley, Reading, MA (1953), p. 83.

[9] G. B. Thomas, Calculus and Analytic Geometry, AddisonWesley, Reading, MA (1953), pp. 497-500.

[10] Ref. [9], pp. 550-553.

[11] C.R.C. Standard Mathematical Tables, Eleventh Edition, C. D. Hodgman, (ed.), Chemical Rubber Publ. Co., Cleveland, $\mathrm{OH}$.

[12] IEEE Electromagnetic Compatibility Society, draft 9, Standard Procedures for the Measurement of Electric and Magnetic Fields from Video Display Terminals (VDTs) from $5 \mathrm{~Hz}$ to $400 \mathrm{kHz}$, July, 1992.

\footnotetext{
About the author: M. Misakian is a physicist in the Electricity Division of NIST. The National Institute of Standards and Technology is an agency of the Technology Administration, U.S. Department of Commerce.
} 